\title{
A lipophilic fluorescent LipidGreen 1-based quantification method for high-throughput screening analysis of intracellular poly-3-hydroxybutyrate
}

\author{
Ji Eun Choi ${ }^{1}$, Hye Young Na ${ }^{1,2}$, Taek Ho Yang ${ }^{3}$, Sung-Keun Rhee ${ }^{2}$ and Jae Kwang Song ${ }^{\text {** }}$
}

\begin{abstract}
Poly-3-hydroxybutyrate (PHB), the most abundant type of polyhydroxyalkanoates (PHA) is synthesized inside a variety of microorganisms as a primary candidate for industrial PHB production. Lipophilic dyes such as Nile red and BODIPY have been used to quantify intracellular PHB, but their uses have often been limited in terms of sensitivity and accuracy. In this study, a newly developed lipophilic fluorescent dye LipidGreen1 was used to quantify intracellular PHB. LipidGreen 1 stained viable colonies by adding the dye into the medium which enabled the effective selection of PHB-positive cells. Furthermore, the fluorescence intensity of LipidGreen1 maintained its fluorescence intensity much longer than that of Nile red. The fluorescence intensities of intracellular PHB stained by LipidGreen1 accurately agreed with PHB contents measured by gas chromatography. In addition, internalization of LipidGreen1 in Escherichia coli cell was not necessary to obtain quantitative measurements. PHB-synthase mutants were differentiated by fluorescence intensities with a good correlation to increased levels of PHB production. These results show that LipidGreen 1 is sensitive and accurate in high-throughput screening of newly isolated and genetically modified bacteria with enhanced PHB production.
\end{abstract}

Keywords: LipidGreen1, Poly-3-hydroxybutyrate, High-throughput screening, Quantitative measurement, Spectrofluorometry, Microtiter plate assay

\section{Introduction}

Polyhydroxyalkanoate (PHA) is a linear polyester which accumulates in various gram-positive and gram-negative bacteria as an intracellular granular material for carbon and energy storage from renewable resources (Snell and Peoples 2009). Bacterial PHA shows a good promise as a biodegradable and biocompatible plastic for packaging and medical applications (Reddy et al. 2013). Therefore, it is considered as a functional substitute for petroleum-based plastics due to thermoplastic and elastomeric properties of its copolymers (Balaji et al. 2013).

\footnotetext{
*Correspondence: ajee@krict.re.kr

${ }^{1}$ Research Center for Bio-based Chemistry, Korea Research Institute of Chemical Technology (KRICT), 141 Gajeong-ro, Yuseong-gu,

Daejeon 305-600, Republic of Korea

Full list of author information is available at the end of the article
}

Poly-3-hydroxybutyrate (PHB), the most abundant type of PHA, can be produced from a variety of sugars. For example, Cupriavidus necator H16 (formerly, Ralstonia eutropha H16) synthesizes PHB from acetyl-CoA through three enzyme reactions (Peoples and Sinskey 1989). PHB production from Cupriavidus necator H16 has been studied in many fields, including the application of non-edible carbon sources into PHB production such as food wastes (Hafuka et al. 2010) and Jatropha oil (Ng et al. 2010). Furthermore, PHB production has been well studied with functional genes of Cupriavidus necator H16 (Budde et al. 2010; Kahar et al. 2004). Recently, many strategies including culture medium manipulations (Khanna and Srivastava 2005; Nath et al. 2008) and genetic modifications (Madison and Huisman 1999; Lim et al. 2002) have been developed to increase PHB production. PHB synthesis in recombinant bacteria is

\section{Springer}


considered to be economically beneficial due to its fast growth rate and high accumulation of PHB up to $90 \%$ of its dry cell weight and thus, has been thoroughly investigated in genetic engineering and culture optimization studies to enhance PHB-productivity (Kim et al. 1992; Slater et al. 1988).

It is necessary to develop better enzymes relevant to PHB biosynthesis and identify high-yield production strains. Thus, a simple and reliable high-throughput method, having the advantage of real time monitoring of cell growth and PHB contents, is needed. Although chromatographic analysis provides the most accurate details relative to PHB quantification and monomer composition, it involves the complex and time-consuming steps such as the extraction and derivatization of PHB. Therefore, it is not suitable for high-throughput measurements of a large number of samples. Nowadays, lipophilic fluorescent dyes such as Nile Red (a benzophenoxazone dye), BODIPY (a boron-dipyrromethene dye) (Cirulis et al. 2012; Tyo et al. 2006; Pinzon et al. 2011) are generally used as a rapid and high-throughput detection method. Nile red has been used to measure PHB contents inside microbial cells with a micro-fluorospectrometer (Schlebusch and Forchhammer 2010; Zuriani et al. 2013) and fluorescence activated cell sorter (FACS) (Kacmar et al. 2005; Tyo et al. 2006). However, the use of Nile red has low sensitivity and poor reliability, when it is used with viable cells growing in a liquid culture medium and entrained in a FACS system (Lee et al. 2013).

LipidGreen 1 is a new small fluorescence probe with an indolin-3-one skeleton, which successfully stained lipid droplets in 3T3-L1 and HepG2 cells and fat deposits in zebrafish (Chun et al. 2013; Lee et al. 2011). LipidGreen 1 could be used to detecting bacterial polyesters including PHB. In this study, we suggested that LipidGreen1 is a powerful tool for rapid and accurate selection of enhanced PHB-producing bacteria with micro-fluorospectrometer. Furthermore, the PHB contents of PHA synthase mutant library could be measured using the high-throughput LipidGreen1 staining method.

\section{Materials and methods}

\section{Plasmids, bacteria and chemicals}

The plasmid pPhaCAB consists of a pBluescript II SK+ backbone (Stratagene, USA) and the PHB biosynthetic gene cluster encoding three genes for type I PHA synthase $(p h a C)$, ketothiolase ( $p h a A)$, and acetoacetyl-CoA reductase (phaB) from Cupriavidus necator H16 (Yang et al. 2010). Escherichia coli XL1-Blue (Stratagene) was transformed with pPhaCAB for expression of the PHA biosynthesis genes. LipidGreen1 was provided by Korea Chemical Bank (KRICT, South Korea; Additional file 1: Fig. S1). LipidGreen1and Nile red stock solutions were prepared by dissolving the dyes in dimethylsulfoxide (DMSO) to a final concentration of $1 \mathrm{mg} / \mathrm{mL}$. PHB powder was purchased from Sigma-Aldrich (USA). Ten milligrams $\mathrm{PHB}$ powder was suspended in $1 \mathrm{~mL}$ water using ultrasonic homogenizer (Sonics and Materials, USA) for 1 min on $20 \%$ amplitude.

\section{Culture conditions}

Recombinant E. coli XL1-Blue transformed with phaCAB, PHB-producing cell, was grown at $37^{\circ} \mathrm{C}$ in Luria-Bertani (LB) medium containing $10 \mathrm{~g} / \mathrm{L}$ tryptone, $5 \mathrm{~g} / \mathrm{L}$ yeast extract, $5 \mathrm{~g} / \mathrm{L} \mathrm{NaCl}$, and $50 \mu \mathrm{g} / \mathrm{mL}$ ampicillin. After $20 \mathrm{~h}$ cultivation in $2 \mathrm{~mL}$ of LB broth, the PHB-producing cells were inoculated into LB medium supplemented with $20 \mathrm{~g} / \mathrm{L}$ glucose and cultured on an incubator at $37^{\circ} \mathrm{C}$ for $20 \mathrm{~h}$ with shaking $(200 \mathrm{rpm})$. For cell viability analysis, the PHB-producing cells were cultivated in $100 \mathrm{~mL}$ LB medium with $20 \mathrm{~g} / \mathrm{L}$ glucose and LipidGreen 1 $(0,0.8$, and $2 \mu \mathrm{g} / \mathrm{mL})$. The cultures were collected every 2 or $3 \mathrm{~h}$ and then optical densities at $600 \mathrm{~nm}$ were measured (Shimadzu, Japan).

\section{Observation of bacterial PHB on an agar plate}

The PHB-producing cells were spread on the agar plate containing LipidGreen 1 at a final concentration of $25 \mu \mathrm{g} /$ $\mathrm{mL}$ and cultured for $20 \mathrm{~h}$ at $37^{\circ} \mathrm{C}$. Accumulation of intracellular PHB was viewed under ultraviolet light $(302 \mathrm{~nm})$. E. coli XL1-Blue, which contains only the pBluescript II SK+ vector (Agilent Technologies, USA), was prepared as a negative control. Subsequently, the PHB-producing and PHB-non-producing cells were scraped from the surface of the agar plates and suspended in $100 \mu \mathrm{L}$ of phosphate-buffered saline (PBS, pH 7.2, $20 \mathrm{mM}$ ). Ten microliters of the suspensions were placed on slide glass and used for microscopic observation by fluorescence microscope (Nikon, Japan) with a green fluorescence filter (Green Excitation 460-500 nm, Emission 510-560 mm).

\section{Measurement of the fluorescence intensity}

The PHB-producing cells were cultivated in $500 \mathrm{~mL} \mathrm{LB}$ medium containing $20 \mathrm{~g} / \mathrm{L}$ glucose. The cells were harvested by centrifugation $\left(3,200 \times g, 4^{\circ} \mathrm{C}\right.$ for $\left.10 \mathrm{~min}\right)$ and resuspended in PBS to yield an optical density at $600 \mathrm{~nm}$ of 2.0. LipidGreen1 was added to the $1 \mathrm{~mL}$ cell suspensions, followed by further incubation for 0.5 and $2 \mathrm{~h}$ in the dark. The final concentration of LipidGreen 1 at $2 \mu \mathrm{g} / \mathrm{mL}$ was used in further experiment. One hundred microliters of the suspensions were immediately transferred into a 96 well black microplate, and the fluorescence intensity was measured within $10 \mathrm{~min}$ with a micro-fluorospectrometer (TECAN, Switzerland) at an excitation wavelength of $450 \mathrm{~nm}$ and emission wavelength of $510 \mathrm{~nm}$. To verify the 
relation between the PHB accumulation contents and fluorescence intensity, $30 \mathrm{~mL}$ of the culture solutions were collected at $2 \mathrm{~h}$ intervals during cell growth and stored at $-70^{\circ} \mathrm{C}$ deep freezer for fluorescence and GC analysis. In addition, the aqueous PHB suspension was serially diluted in water and then incubated with LipidGreen1 for $30 \mathrm{~min}$ in black microtubes followed by measurement of fluorescence intensities. Furthermore, Nile red was added to the $1 \mathrm{~mL}$ cell suspensions to give a final concentration of $2 \mu \mathrm{g} / \mathrm{mL}$, and then fluorescence intensities were measured at 540 and $570 \mathrm{~nm}$ for the excitation and emission wavelengths, respectively.

\section{Comparison of fluorescence intensity between intact and lysed cells}

The PHB-producing cells grown in the $50 \mathrm{~mL}$ medium were collected by centrifugation $\left(4^{\circ} \mathrm{C}\right.$ at $3,200 \times g$ for $10 \mathrm{~min}$ ) to an optical density at 600 of 4.0. Half of the cell suspension in PBS buffer was disrupted with the ultrasonic homogenizer, while the remaining suspension was left on ice as intact cells. One milliliter each of disrupted and intact cell suspension was moved into a black microtube followed by the addition of LipidGreen 1 . The mixtures were incubated for 0.5 and $2 \mathrm{~h}$ and immediately transferred into a black 96-well microplate to measure the fluorescence intensity.

\section{PHB quantification by gas chromatography (GC)}

The PHB polymer content was determined by GC analysis as previously described (Yang et al. 2010). Briefly, PHB-producing cells were washed twice with PBS buffer and dried at $65^{\circ} \mathrm{C}$ in an oven with a final dry pellet weight of $0.03 \mathrm{~g}$. The dry matter was subjected to methanolysis in the presence of $1 \mathrm{~mL}$ PHA solution containing $0.8 \%$ (wt/vol) Benzoic acid, 3\% (vol/vol) sulfuric acid, 97\% (vol/ vol) methanol and $2 \mathrm{~mL}$ of chloroform. Following $6 \mathrm{~h}$ of incubation at $100^{\circ} \mathrm{C}$, the polymer solutions dissolved in chloroform were precipitated and separated with chilled deionized water. The PHB contents were analyzed by GC (6890N GC system, Agilent Technologies) equipped with a fused silica capillary column (SPBTM-5, $30 \mathrm{~m} \times 0.32 \mathrm{~mm}$ ID, $0.25 \mu \mathrm{m}$ film; Supelco, USA) using benzoic acid as an internal standard.

\section{Construction and screening of a phaC mutant library}

Random mutagenesis was performed by error-prone PCR with GeneMorph II Random mutagenesis kit (Stratagene) following the manufacturer's instructions. Briefly, to introduce random mutations into the phaC gene, forward and reverse primers (ReCMutF; 5'-GATCCCCCGGGCAAGTACC-3, ReCMutR; 5'-GGGAACCTGCAGGCCTGC-3') were designed based on the nucleotide sequences outside of the structural gene.
Each PCR contained $30 \mathrm{ng}$ of the pPhaCAB plasmid as the initial template, $250 \mathrm{ng}$ of each primer, $200 \mu \mathrm{M}$ of each dNTP, and $5 \mathrm{U}$ of Taq DNA polymerase in Taq DNA polymerase reaction buffer. The PCR started with a denaturation step at $95^{\circ} \mathrm{C}$ for $30 \mathrm{~s}$, followed by 25 cycles of amplification $\left(30 \mathrm{~s}\right.$ at $95^{\circ} \mathrm{C}, 30 \mathrm{~s}$ at $55^{\circ} \mathrm{C}$, and $3 \mathrm{~min}$ at $72^{\circ} \mathrm{C}$ ), and a final extension step at $72^{\circ} \mathrm{C}$ for $10 \mathrm{~min}$. The PCR products purified with the QIAquick PCR Purification Kit (Qiagen, USA) were digested with SmaI and SbfI and subjected to preparative electrophoresis in a $0.8 \%$ agarose gel. The approximately $1.8-\mathrm{kb}$ PCR fragments were ligated into the same restriction sites of the $\mathrm{pPhaCAB}$ vector, and then the ligates were transformed into E. coli XL1-Blue. Each mutant clone was grown in a deep-well microplate containing $800 \mu \mathrm{L}$ of the $\mathrm{LB}$ medium containing $20 \mathrm{~g} / \mathrm{L}$ glucose for $20 \mathrm{~h}$, of which $100 \mu \mathrm{L}$ were transferred to a black microplate to measure its fluorescence intensity by adding LipidGreen 1 . Determination of the cellular PHB content by GC was done with selected clones that showed relatively higher or lower fluorescence intensities than that of the wild type.

\section{Results}

LipidGreen 1 staining of PHB-producing bacterial cells on agar plates

LipidGreen1, a novel fluorescent dye previously used to stain neutral lipids and fat deposits in eukaryotic cells and tissues (Lee et al. 2011), has a unique chemical structure consisting of an indoline-3-one skeleton. The core skeleton of LipidGreen1 is markedly different from a benzophenoxazine skeleton of the representative lipophilic fluorescent dye, Nile red (Additional file 1: Fig. S1). We examined LipidGreen 1 staining for PHB accumulated inside bacterial cells. When the $E$. coli cells harboring $\mathrm{pPhaCAB}$ were grown for $20 \mathrm{~h}$ on agar plates containing $25 \mu \mathrm{g} / \mathrm{mL}$ LipidGreen1, they exhibited a far stronger fluorescence than the E. coli cells harboring pBluescript II SK(+) vector (Fig. 1a). The E. coli cells stained with LipidGreen1 on agar plates were collected, suspended in PBS buffer ( $\mathrm{pH}$ 7.2), and observed with fluorescence microscopy (Fig. 1b). The strong fluorescence of the PHB-producing E. coli cells also indicated that the LipidGreen1 was capable of detecting the PHB accumulation of $E$. coli cell grown on agar plates and that its fluorescent signal was maintained for quite a long time.

\section{LipidGreen 1 staining of PHB-producing bacterial cells in liquid medium}

After PHB-producing cells were grown in the medium, LipidGreen 1 to a final concentration of $2 \mu \mathrm{g} / \mathrm{mL}$ was added to the cell suspension and then incubated for 0.5 and $2 \mathrm{~h}$. The fluorescence intensity of LipidGreen 1 was 740 in PHB-producing cells (Fig. 2), whereas that of the 

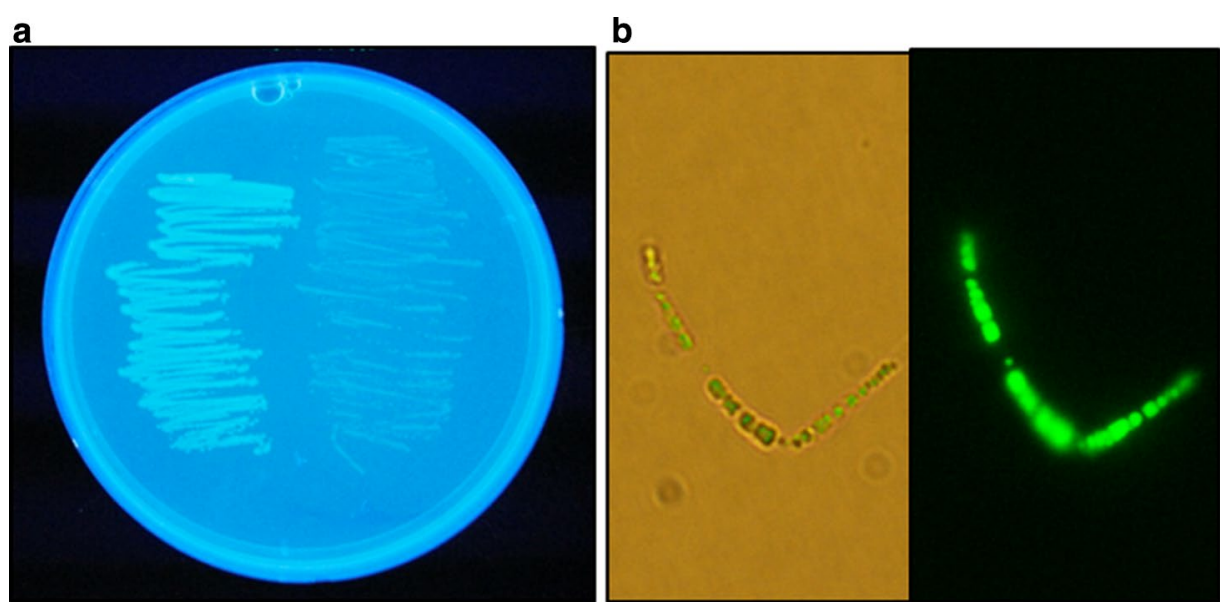

Fig. 1 Fluorescence of PHB-producing cells grown on a LipidGreen-containing agar plate. a Fluorescence staining of PHB-producing and PHBnonproducing cells on an agar plate. E. coli harboring pPhaCAB (left side of the plate) had bright fluorescence under UV light (302 nm) in contrast to E. coli containing pBluescript II SK+ vector (right side of the plate) exhibiting a much weaker fluorescence than above. b Microscopic observation of E. coli stained by LipidGreen1 on agar plates. The cell suspension in PBS buffer was placed on a glass slide to obtain optic (left) and fluorescent (right) images. The images were viewed under the fluorescence microscope ( $\times 1,000$ amplification) with a green fluorescence filter.

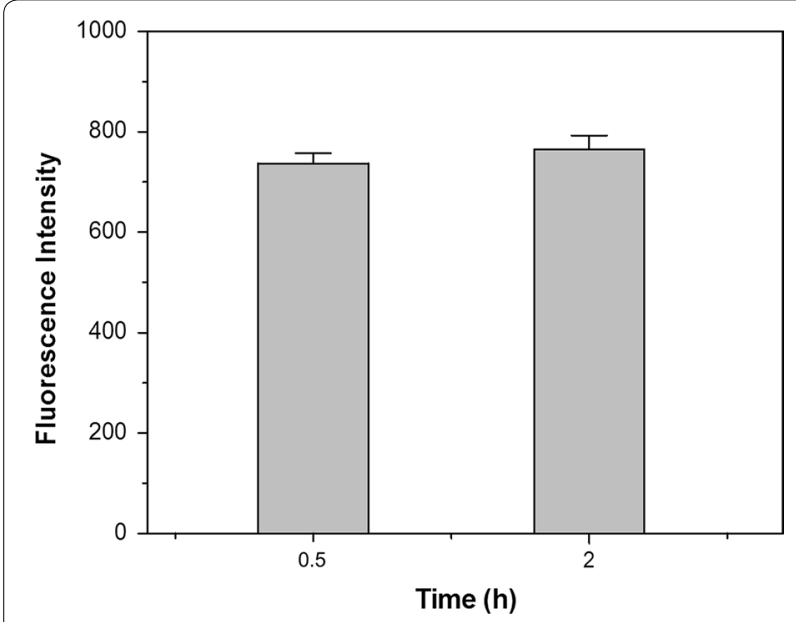

Fig. 2 Comparison of fluorescence intensity for LipidGreen1 at different time points. A PHB-producing cell suspension at $\mathrm{OD}_{600} 2.0$ was stained with LipidGreen 1 at a final concentration of $2.0 \mu \mathrm{g} / \mathrm{ml}$. Gray bars are fluorescence intensities for incubation times of 0.5 and $2 \mathrm{~h}$ after adding LipidGreen 1, respectively. The data shown are the means with standard deviation (error bars) from three independent experiments.

E. coli cells containing none of PHB was 248 (data not shown). In particular, the fluorescence intensity of LipidGreen 1 was stably maintained for $2 \mathrm{~h}$. On the other hand, the fluorescence intensity of Nile red as a representative fluorescent lipophilic dye was significantly decreased only after $1 \mathrm{~h}$ incubation with the PHB-producing cells (Additional file 1: Fig. S2). We also found out that LipidGreen1 was not harmful to the cell growth (Additional file 1: Fig. S3-A). When the PHB-producing E. coli was grown in the LB medium containing $20 \mathrm{~g} / \mathrm{L}$ glucose and LipidGreen 1 up to the final concentration of $2 \mu \mathrm{g} / \mathrm{mL}$, the cell growth in the presence of LipidGreen 1 ( 0.8 and $2.0 \mu \mathrm{g} / \mathrm{mL}$ ) was similar to the cell growth in the absence of LipidGreen1. Also, PHB-producing cells showed similar growth between Nile red containing $(0,0.8,2 \mu \mathrm{g} / \mathrm{mL})$ and non-containing $\mathrm{PHB}$-producing medium (Additional file 1: Fig. S3-B). Differences in the fluorescence intensity between membrane-disrupted and non-disrupted cells were measured to assess the LipidGreen permeability to cell membrane. Ultrasonication to $E$. coli cells caused an increase in fluorescence intensity (Fig. 3). However, regardless of whether the cells were disrupted or not, the prolonged incubation for 0.5 and $2 \mathrm{~h}$ after adding LipidGreen 1 did not influence the fluorescence intensity.

\section{Quantitative correlation between fluorescence intensity and PHB contents}

The feasibility of LipidGreen1 staining for the quantitative measurement of $\mathrm{PHB}$ contents was examined using the aqueous $\mathrm{PHB}$ suspension and the PHB-producing cells. The fluorescence intensity of PHB suspension ranging from 0.15 to $5 \mathrm{~g} / \mathrm{L}$ increased linearly with a high correlation coefficient $\left(\mathrm{R}^{2}=0.96\right)$ (Fig. 4a). The cell suspensions prepared from a different amount of PHB-producing $E$. coli cells were analyzed both by LipidGreen1 staining and by GC-based quantification of the purified cellular PHB. There was a good agreement $\left(R^{2}=0.96\right)$ between the fluorescence intensities and the amount of PHB measured by GC (Fig. 4b). The fluorescence 


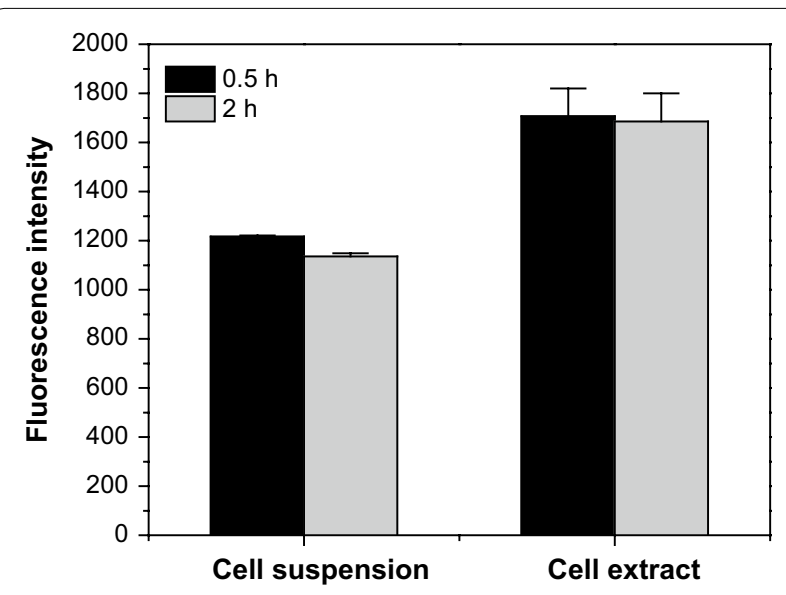

Fig. 3 Differences in fluorescence intensity between cell suspensions and cell extracts. Fully grown PHB-producing cells were resuspended into PBS to an $\mathrm{OD}_{600}$ of 4.0. Fluorescence intensity was estimated using non-treated cells and lysed cells by ultrasonication after prolonged incubation for 0.5 and $2 \mathrm{~h}$.

intensity by Nile red staining for the purified cellular PHB had a lower relationship $\left(\mathrm{R}^{2}=0.76\right)$ to the $\mathrm{GC}$ based amount of PHB (Fig. 4c).

\section{High-throughput screening of PHB contents in a phaC mutant library}

The PHA synthase gene (phaC) in the PHB biosynthesis gene cluster was randomly mutated by error-prone PCR. A set of $E$. coli clones, each containing a variant of the phaC gene, was selected randomly and used to measure the fluorescence intensity of intracellular PHB. When the mutant clones were cultivated in deep-well plates for $20 \mathrm{~h}$ and stained with LipidGreen1, the fluorescence intensities of $p h a C$ variants widely differed from wild type $p h a C$, ranging from 500 to 3,000 , which obviously was due to the difference in their PHB-synthesizing ability (Fig. 5). When compared to the wild-type phaC, about $60 \%$ of the mutant clones had lower fluorescence intensities. In contrast, $25 \%$ of the phaC variants among 60 mutant clones were observed to have higher fluorescence intensities than that of the wild type, and in particular, $2 \%$ of the phaC variants found had a more than twofold increase in fluorescence intensity. To verify whether the fluorescence intensity of bacterial PHB stained by LipidGreen 1 agreed with the intracellular PHB contents, we selected mutant clones that exhibited increased or decreased fluorescence intensities compared to wild type $p h a C$ and analyzed the PHB contents with GC. PHB accumulations were measured for two clones that showed twofold higher fluorescence intensities (M11 and M54) and for three clones that showed lower fluorescence intensities (M3, M21 and M43) than that of the wild type. Consequently, the
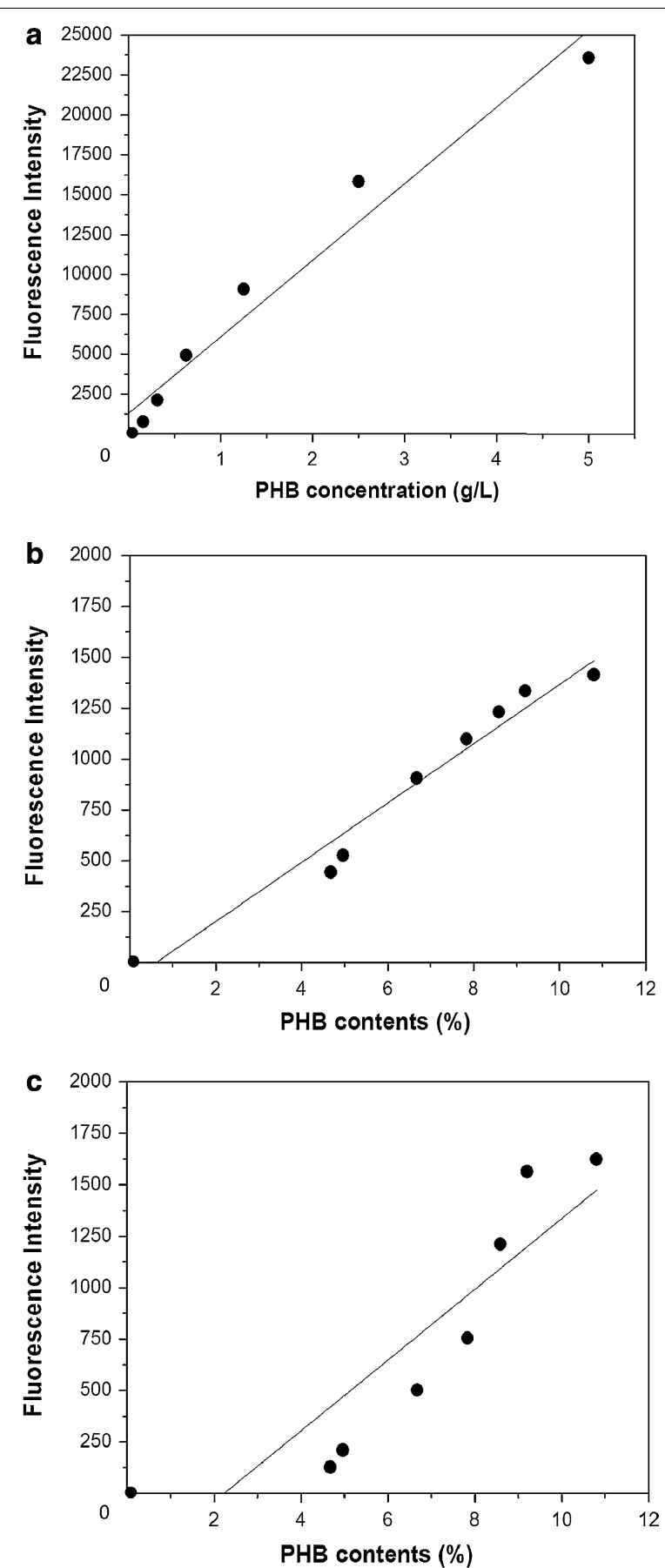

Fig. 4 Fluorescence intensities of PHB powder and intracellular PHB granules. To exam the correlation between PHB concentration and fluorescence intensity, different concentrations of aqueous PHB suspension were incubated with $2 \mathrm{\mu g} / \mathrm{mL}$ LipidGreen 1 for $30 \mathrm{~min}$, and then the fluorescence intensities were measured at $450 \mathrm{~nm}$ excitation and $510 \mathrm{~nm}$ emission wavelengths, respectively (a). On the other hands, intracellular PHB contents were measured by collecting PHBproducing cells every 2 or $3 \mathrm{~h}$ during cultivation. The fluorescence intensities were measured with $2.0 \mathrm{\mu g} / \mathrm{ml}$ of LipidGreen 1 (b) and Nile red $(\mathbf{c})$. The PHB contents of the cells at different times were analyzed by GC. The solid line indicates the regression line. 


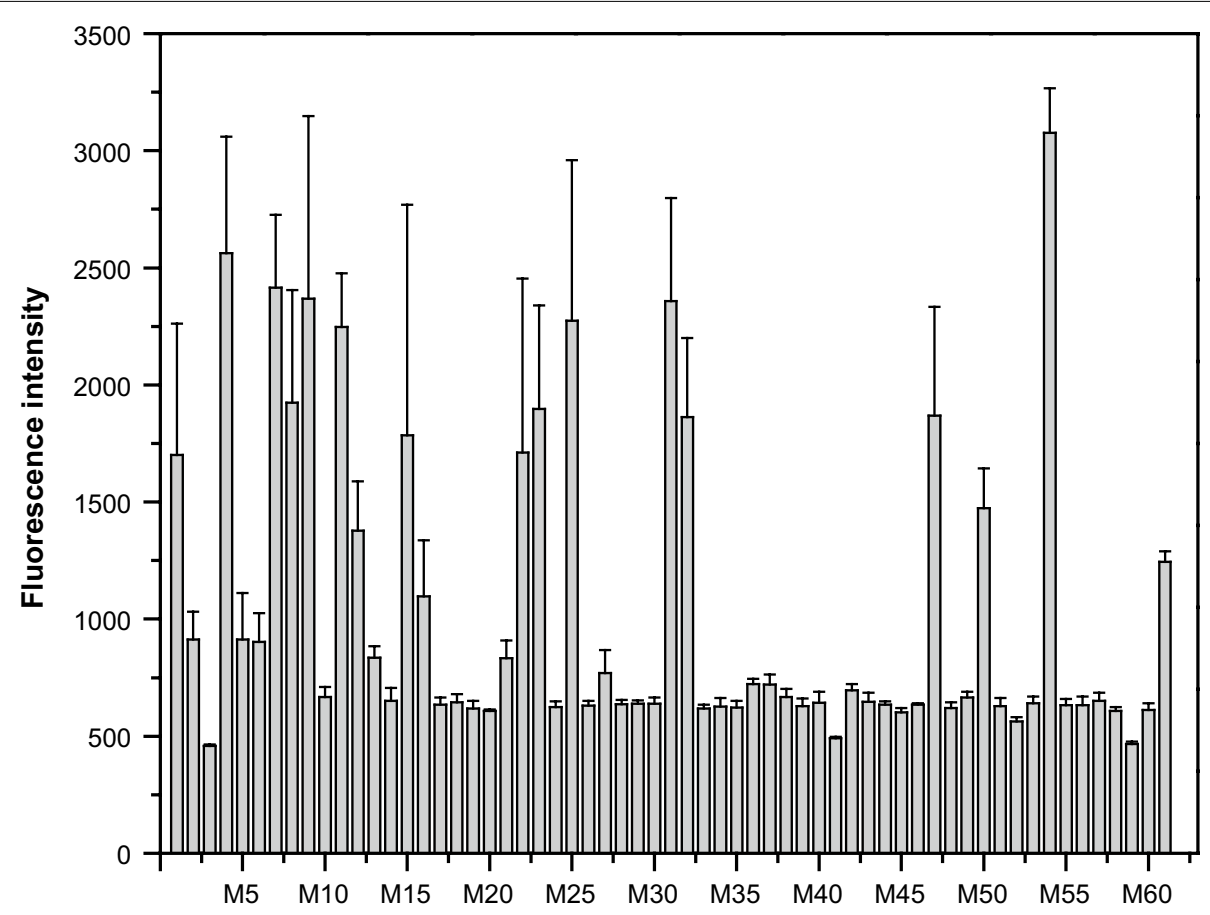

Fig. 5 Fluorescence intensities of the mutant library, which consisted of a pool of phaC genes with PCR-introduced random mutations. Every single mutant clone was inoculated in a 96-deep well plate and cultivated for $20 \mathrm{~h}$ in $800 \mu \mathrm{l} \mathrm{of} \mathrm{LB}$ medium with $20 \mathrm{~g} / \mathrm{L}$ glucose. Fluorescence intensities were monitored after transferring $100 \mu$ of culture solution into a black microplate. The x axis represents the names of the mutants.

mutant clone M54 showing 2.5 times higher fluorescence intensity than wild type phaC accumulated about 2.5 times more PHB than that of the wild type phaC (Additional file 1: Table S1). The fluorescence intensity of clone M11 producing double amount of PHB contents than wild type phaC also showed twice higher values than that of wild type. In contrast, mutant M43 with half the fluorescence intensity of wild type phaC had about $10 \%$ of the normal PHB accumulation found in wild type, showing a lower detection limit.

\section{Discussion}

It has been often noted that intensities of fluorescence signal by lipophilic dyes were not directly proportional to the amount of lipids (O'Rourke et al. 2009). For example, Nile red poorly stained high fat-containing tissues of $C$. elegans such as germline, eggs, and hypodermis. Furthermore, Nile red and BODIPY were stained the lysosomerelated organelles rather than the major C. elegans fat storage compartment. In the previous studies on LipidGreen 1 and its derivatives, LipidGreen1 was capable of a brighter and less non-specific staining for the detection of neutral lipids and fat deposits compared to the commercially available Nile red and BODIPY ${ }^{\circledR}$ 493/503 (Lee et al. 2011; Chun et al. 2013). Therefore, we attempted to examine a possibility for the more reliable detection of bacterial PHB, one of the promising biodegradable plastics, using the lipophilic fluorescent LipidGreen1.

As shown in Fig. 1, the PHB-producing cells on agar plates containing the fluorescent LipidGreen 1 showed the green emission fluorescence under UV irradiation. The result indicated that the LipidGreen 1 could enter a bacterial cell probably by diffusion across the cell wall and the inner and outer membranes to the cytoplasm where it subsequently bound to the PHB granules. Furthermore, the LipidGreen1 was not harmful for the bacterial growth on agar plates (Fig. 1) and in liquid medium (Additional file 1: Fig. S3), and thus could be used from the beginning of the bacterial culture. The fluorescent Nile red could be also used to detect PHB in growing bacterial cells by directly including the DMSO-based dye solution in the culture medium (Spiekermann et al. 1999).

The major limitation of Nile red is that the fluorescence intensity gradually diminishes after adding the dye. A significant decrease in fluorescence intensity over $10 \mathrm{~min}$ was observed when $0.5 \%$ of soybean oil distributed in $0.3 \%$ Tween 80 solution was stained with $40 \mu \mathrm{l}$ of $250 \mu \mathrm{g} /$ $\mathrm{ml}$ Nile red (Montalbo-Lomboy et al. 2014). In addition, the maximum fluorescence intensity of oleaginous yeasts was reached between 1 and $5 \mathrm{~min}$ after adding Nile red and slowly faded after 5 min (Kimura et al. 2004). In 
this study, the cell suspension incubated with $0.8 \mu \mathrm{g} /$ $\mathrm{mL}$ Nile red showed high potency of photo-quenching (Additional file 1: Fig. S2). The fluorescence intensities decreased after $1 \mathrm{~h}$-incubation in cell resuspension even though the fluorescence intensities were almost absent after $19 \mathrm{~h}$. The reason why fluorescence intensity of Nile red steadily decreased during incubation with oil or polyester-accumulating cells was due to its hydrophobic property, which maintained its fluorescence only in nonpolar solvents (Greenspan and Fowler 1985). However, in the case of LipidGreen1, the fluorescence intensities were steadily maintained with only slight differences between the time points at 0.5 and $2 \mathrm{~h}$ (Fig. 2). Calculated hydrophobicity (cLogP values) result using Chemdraw program agreed with above data, which hydrophobicity of Nile red $(\mathrm{cLogP}=4.6)$ was higher than that of LipidGreen1 $(\mathrm{cLogP}=4.1)$. The lower hydrophobicity of LipidGreen1 was supposed to contribute maintaining a good fluorescence intensity in aqueous solution by distributed the dye in aqueous solution without loss of its fluorescence intensity. Therefore, LipidGreen1 is a good candidate for a fluorescent probe to monitor PHB accumulation in situ.

Due to the unique and asymmetric lipid composition of bacteria, the outer membrane is fairly impermeable to hydrophobic compounds and moderately so to hydrophilic compounds. Thus, lipophilic dyes such as Nile red and FITC have limited membrane permeability (Herrera et al. 2002). In this study, the fluorescence intensity of the membrane disrupted cells stained with LipidGreen1 was 1.5 times higher than that of intact cells, suggesting insufficient accessibility of the dye to the intracellular PHB (Fig. 3). Previously, the addition of salts or solvents enabling adequate access for the fluorescent molecules to the intracellular PHB such as sucrose and DMSO was suggested to improve the fluorescence intensity and sensitivity (Tyo et al. 2006; Lee et al. 2013; Chen et al. 2009). Nevertheless, the increment of fluorescence intensities was proportional with the PHB concentration to the GC measurement, indicating that the quantitative measurement of intracellular PHB is possible without permeabilization of LipidGreen1 (Fig. 4b). On the contrary, Nile red showed lower correlation coefficient value and specificity to PHB than LipidGreen1 in this experiment (Fig. 4c). We supposed that the exclusion of internalized Nile red in $E$. coli cells lowered the sensitivity and specificity to PHB. Therefore, in term of necessity for internalization, LipidGreen1 had an advantage over Nile red because LipidGreen 1 could omit the membrane-permeability procedures for quantitative intracellular PHB measurement.

The screening of mutant library of PHB polymerase in C. necator resulted in no clones with a distinctly higher PHB accumulation compared to wild type (Taguchi et al. 2001). PHB accumulation in all the mutant clones was lower than that of the wild type clone, suggesting that the wild-type PHB polymerase is highly optimized for PHB accumulation defined as the 'fitness landscape model' (Taguchi et al. 2001). In the same manner, about 200,000 mutants of PHA synthase gene from Aeromonas punctata were screened and only five mutants with enhanced fluorescence were isolated (Amara et al. 2002). In this study, most of the mutant clones had much lower or similar fluorescence intensities compared to the wild type consistent with 'fitness landscape model. Despite of that, $2 \%$ out of total mutant clones showed the distinctively improved fluorescence intensities (Fig. 5). The increased fluorescence intensities of mutant clones agreed with the increment of PHB amount measured by GC. Thus, high-throughput screening of PHB synthase mutant library was available using LipidGreen1 through 96-well microtiter plate-based assay. In addition the PHB amount can be measured with a preparation time of less than $10 \mathrm{~min}$ and only a small sample volume $(100-200 \mu \mathrm{L})$.

In conclusion, quantitative measurement of intracellular PHB with a new fluorescent dye LipidGreen1 was confirmed that PHB accumulation can be visualized in viable colonies by LipidGreen1, providing tools to distinguish between PHB-producing and nonproducing cells. Moreover, LipidGreen1 was highly effective in quantifying PHB by fluorescence measurement because of its prolonged sustainability providing better accuracy and sensitivity than Nile red. A bacterial mutant with enhanced PHB production can be distinguished from randomly mixed samples such as random mutagenesis by error-prone PCR in a high-throughput manner using LipidGreen1. LipidGreen1 could be developed into a commercial kit that rapidly determines the PHB contents, achieving real time monitoring for mass production.

\section{Additional file}

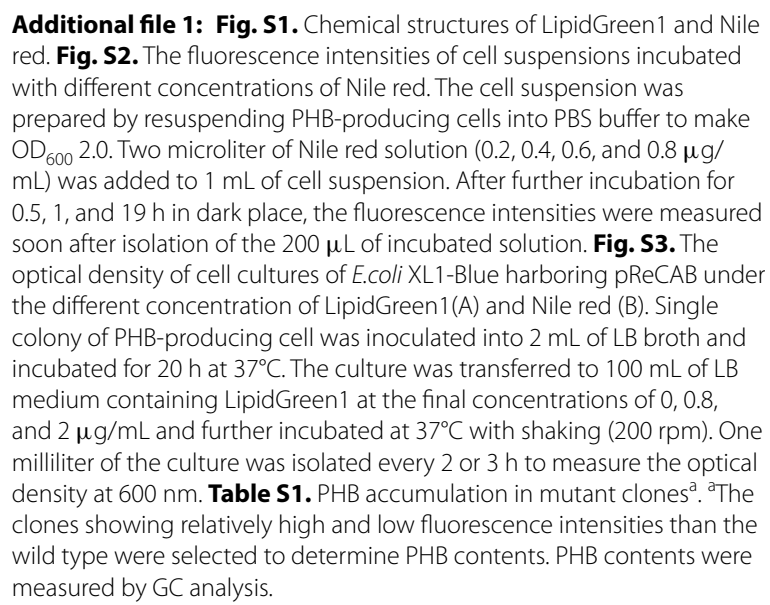




\section{Author details}

${ }^{1}$ Research Center for Bio-based Chemistry, Korea Research Institute of Chemical Technology (KRICT), 141 Gajeong-ro, Yuseong-gu, Daejeon 305-600, Republic of Korea. ${ }^{2}$ Department of Microbiology, Chungbuk National University, Chungdae-ro 1, Seowon-gu, Cheongju, Chungbuk 362-763, Republic of Korea. ${ }^{3}$ GenoFocus, 65 Techno 1-ro, Yusung-gu, Daejeon 305-509, Republic of Korea.

\section{Acknowledgements}

This work was supported by the Intelligent Synthetic Biology Center of Global Frontier Project (NRF-2014M3A6A8065315) through the National Research Foundation of Korea (NRF), funded by the Ministry of Science, ICT, and Future Planning. We would like to thank Dr. Jinhee An and Dr. Myung-Ae Bae, Bioorganic Division, Korea Research Institute of Chemical Technology, for providing LipidGreen 1

\section{Compliance with ethical guidelines}

\section{Competing interests}

The authors declare that they have no competing interests.

Received: 13 July 2015 Accepted: 17 July 2015

Published online: 09 August 2015

\section{References}

Amara AA, Steinbüchel A, Rehm BHA (2002) In vivo evolution of the Aeromonas punctata polyhydroxyalkanoate (PHA) synthase: isolation and characterization of modified PHA synthases with enhanced activity. Appl Microbiol Biotechnol 59:477-482

Balaji S, Gopi K, Muthuvelan B (2013) A review on production of poly $\beta$ hydroxybutyrates from cyanobacteria for the production of bio plastics. Algal Res 2:278-285

Budde CF, Mahan AE, Lu J, Rha C, Sinskey AJ (2010) Roles of multiple acetoacetyl coenzyme A reductases in polyhydroxybutyrate biosynthesis in Ralstonia eutropha H16. J Bacteriol 192:5319-5328

Chen W, Zhang C, Song L, Sommerfeld M, Hu Q (2009) A high throughput Nile red method for quantitative measurement of neutral lipids in microalgae. J Microbiol Methods 77:41-47

Chun HS, Jeon JH, Pagire HS, Lee JH, Chung HC, Park MJ et al (2013) Synthesis of LipidGreen2 and its application in lipid and fatty liver imaging. Mol Biosyst 9:630-633

Cirulis JT, Strasser BC, Scott JA, Ross GM (2012) Optimization of staining conditions for microalgae with three lipophilic dyes to reduce precipitation and fluorescence variability. Cytom A 81:618-626

Greenspan P, Fowler SD (1985) Spectrofluorometric studies of the lipid probe, nile red. J Lipid Res 26:781-789

Hafuka A, Sakaida K, Satoh H, Takahashi M, Watanabe Y, Okabe S (2010) Effect of feeding regimens on polyhydroxybutyrate production from food wastes by Cupriavidus necator. Bioresour Technol 102:3551-3553

Herrera G, Martinez A, Blanco M, O'Connor JE (2002) Assessment of Escherichia coli $\mathrm{B}$ with enhanced permeability to fluorochromes for flow cytometric assays of bacterial cell function. Cytom A 49:62-69

Kacmar J, Carlson R, Balogh SJ, Srienc F (2005) Staining and quantification of poly-3-hydroxybutyrate in Saccharomyces cerevisiae and Cupriavidus necator cell populations using automated flow cytometry. Cytom A 69:27-35

Kahar P, Tsuge T, Taguchi K, Doi Y (2004) High yield production of polyhydroxyalkanoates from soybean oil by Ralstonia eutropha and its recombinant strain. Polym Degrad Stab 83:79-86

Khanna S, Srivastava AK (2005) Statistical media optimization studies for growth and PHB production by Ralstonia eutropha. Process Biochem 40:2173-2182

Kim BS, Lee SY, Chang HN (1992) Production of poly- $\beta$-hydroxybutyrate by fedbatch culture of recombinant Escherichia coli. Biotechnol Lett 9:811-816

Kimura K, Yamaoka M, Kamisaka Y (2004) Rapid estimation of lipids in oleaginous fungi and yeasts using Nile red fluorescence. J Microbiol Methods $56: 331-338$
Lee JH, So JH, Jeon JH, Choi EB, Lee YR, Chang YT et al (2011) Synthesis of a new fluorescent small molecule probe and its use for in vivo lipid imaging. Chem Commun 47:7500-7502

Lee JH, Lee SH, Yim SS, Kang KH, Lee SY, Park SJ et al (2013) Quantified highthroughput screening of Escherichia coli producing poly(3-hydroxybutyrate) based on FACS. Appl Biochem Biotechnol 170:1767-1779

Lim SJ, Jung YM, Shin HD, Lee YH (2002) Amplification of the NADPH-related genes $z w f$ and gnd for the oddball biosynthesis of PHB in an E. coli transformant harboring a cloned phbCAB operon. J Biosci Bioeng 93:543-549

Madison LL, Huisman GW (1999) Metabolic engineering of poly(3-hydroxyalkanoates): from DNA to plastic. Microbiol Mol Biol Rev 63:21-53

Montalbo-Lomboy M, Kantekin MN, Wang T (2014) Lipid estimation of surfactant-extracted microalgae oil using Nile red. J Am Oil Chem Soc 91:665-680

Nath A, Dixit M, Bandiya A, Chavda S, Desai AJ (2008) Enhanced PHB production and scale up studies using cheese whey in fed batch culture of Methylobacterium sp. ZP24. Bioresour Technol 99:5749-5755

Ng KS, Ooi WY, Goh LK, Shenbagarathai R, Sudesh K (2010) Evaluation of jatropha oil to produce poly(3-hydroxybutyrate) by Cupriavidus necator $\mathrm{H} 16$. Polym Degrad Stab 95:1365-1369

O'Rourke EJ, Soukas AA, Carr CE, Ruvkun G (2009) C. elegans major fats are stored in vesicles distinct from lysosome-related organelles. Cell Metab 10:430-435

Peoples OP, Sinskey AJ (1989) Poly-beta-hydroxybutyrate (PHB) biosynthesis in Alcaligenes eutrophus H16. Identification and characterization of the PHB polymerase gene (phbC). J Biol Chem 264:15298-15303

Pinzon NM, Aukema KG, Gralnick JA, Wackett LP (2011) Nile red detection of bacterial hydrocarbons and ketones in a high-throughput format. $\mathrm{mBio}$ 2:e00109-e00111

Reddy MM, Vivekanandhan S, Misra M, Bhatia SK, Mohanty AK (2013) Bio based plastics and bionanocomposites: current status and future opportunities. Prog Polym Sci 38:1653-1689

Schlebusch M, Forchhammer K (2010) Requirement of the nitrogen starvationinduced protein Sll0783 for polyhydroxybutyrate accumulation in Synechocystis sp. strain PCC 6803. Appl Environ Microbiol 76:6101-6107

Slater SC, Voige WH, Dennis DE (1988) Cloning and expression in Escherichia coli of the Alcaligenes eutrophus $\mathrm{H} 16$ poly- $\beta$-hydroxybutyrate biosynthetic pathway. J Bacteriol 170:4431-4436

Snell KD, Peoples OP (2009) PHA bioplastic: a value-added coproduct for biomass biorefineries. Biofuels Bioprod Bioref 3:456-467

Spiekermann P, Rehm BH, Kalscheuer R, Baumeister D, Steinbüchel A (1999) A sensitive, viable-colony staining method using Nile red for direct screening of bacteria that accumulate polyhydroxyalkanoic acids and other lipid storage compounds. Arch Microbiol 171:73-80

Taguchi S, Maehara A, Takase K, Nakahara M, Nakamura H, Doi Y (2001) Analysis of mutational effects of a polyhydroxybutyrate (PHB) polymerase on bacterial PHB accumulation using an in vivo assay system. FEMS Microbiol Lett 198:65-71

Tyo KE, Zhou H, Stephanopoulos GN (2006) High-throughput screen for poly3-hydroxybutyrate in Escherichia coli and Synechocystis sp. strain PCC6803. Appl Environ Microbiol 72:3412-3417

Yang TH, Kim TW, Kang HO, Lee SH, Lee EJ, Lim SC et al (2010) Biosynthesis of polylactic acid and its copolymers using evolved propionate CoA transferase and PHA synthase. Biotechnol Bioeng 105:150-160

Zuriani R, Vigneswari S, Azizan MNM, Majid MIA, Amirul AA (2013) A high throughput Nile red fluorescence method for rapid quantification of intracellular bacterial polyhydroxyalkanoates. Biotechnol Bioprocess Eng $18: 472-478$ 